\title{
Ion selective optodes: from the bulk to the nanoscale
}

\author{
Xiaojiang Xie • Eric Bakker
}

Received: 12 October 2014 / Revised: 8 December 2014 / Accepted: 13 December 2014 / Published online: 22 January 2015

(C) Springer-Verlag Berlin Heidelberg 2015

\begin{abstract}
This review describes recent advances in the miniaturization of ion selective optodes into microscale and nanoscale sensors. The topics include a comparison between filmbased and miniaturized ion optodes, equilibrium and exhaustive detection modes, recent preparation methodologies and applications of microscale and nanoscale ion optodes, criteria for the design of optode sensors, and other future perspectives.
\end{abstract}

Keywords Nanosensor · Ion selective optodes ·

Microsphere $\cdot$ Nanosphere $\cdot$ Optical sensor

\section{Introduction}

The quantification and monitoring of ionic species is a ubiquitous challenge encountered in but not limited to environmental science, biochemical research, food chemistry and clinical diagnostics [1-5]. Among other techniques, such as electrochemical methods, atomic (flame) emission spectrometry (AES), mass spectroscopy, atomic absorption spectrometry (AAS), fluorimetry, and capillary electrophoresis (CE), ion selective optical sensors provide a number of advantages with low cost and high versatility and compatibility with other assays [6-13]. A variety of optical ion sensors have been discovered, including fluorescent molecular probes, surface modified nanomaterials, and biologically expressed proteins [14-19].

Published in the topical collection Direct Optical Detection with guest editors Guenter Gauglitz and Jiri Homola.

X. Xie $(\bowtie) \cdot$ E. Bakker $(\square)$

Department of Inorganic and Analytical Chemistry, University of

Geneva, Quai E.-Ansermet 30, 1211 Geneva, Switzerland

e-mail: xiaojiang.xie@unige.ch

e-mail: eric.bakker@unige.ch
It is unlikely that one type of sensor will dominate the entire field because each has its advantages and limitations. For instance, when applied to intracellular imaging, many synthetic molecular probes may leach, sequester, and have a limited loading concentration, but they are bright and easy to handle. The delivery can be noninvasive as well. Autofluorescent protein-based genetically encoded indicators can be expressed in vivo at specific locations. In contrast, molecular probes cannot differentiate cell types or cellular compartments. However, the varieties of the fluorophores for autofluorescent proteins are quite limited and their brightness needs improvement. Functionalized nanomaterials can overcome some of the disadvantages mentioned above, but delivery of large nanoparticles can be quite invasive and their biotoxicity/biocompatibility can be problematic.

Ionophore-based ion selective optical sensors, also called ion selective optodes (sometimes simply optodes) take advantage of the excellent selectivity of readily available ionophores (ion receptors). The working principle is shown in Scheme 1. Compared with other types of optical ion sensors, such as organic fluorescent molecular probes, the recognition of the analyte does not directly result in an optical signal change from a reporter covalently attached to the binding moiety. Instead, the recognition part and the optical reporter are two separate entities. An ionophore is used for the ion recognition, whereas a lipophilic $\mathrm{pH}$ indicator (also called chromoionophore) acts as the optical reporter. As the analyte level in the sample increases, the hydrogen ion concentration in the sensor decreases on the basis of ion-exchange. Therefore, the analyte activity can be detected indirectly by monitoring the hydrogen ion level in the sensor.

Ion selective optodes are versatile optical sensing tools that emerged from the field of polymeric membrane ion selective electrodes (ISEs) and are attractive for their sensor specificity because of the selective ionophores directly adopted from their ISEs counterparts. Moreover, the use of a separate 
Scheme 1 Schematic illustration of how an ion selective optode containing a lipophilic $\mathrm{pH}$ indicator (chromoionophore), an ion receptor (ionophore) and an ion exchanger $\left(\mathrm{R}^{-}\right)$works with constant $\mathrm{pH}$ condition. The three sensing components are embedded in an organic hydrophobic environment that remains electrically neutral. Increasing the $\mathrm{M}^{+}$level will result in a decrease in the protonation degree of the chromoionophore (and vice versa), which can be optically detected

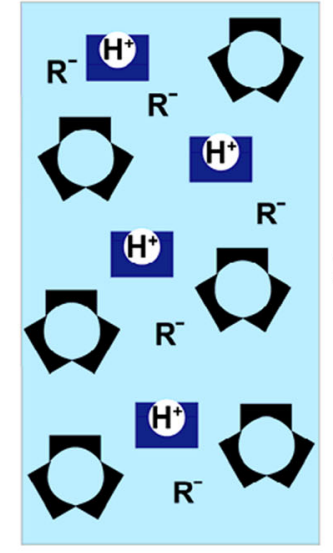

Organic Sensing Phase
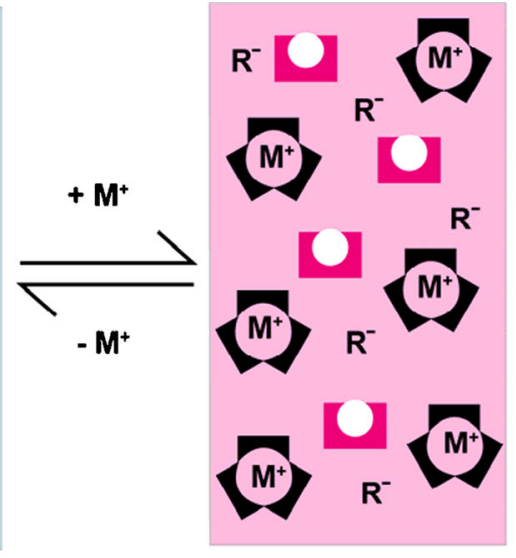

Organic Sensing Phase
LEGEND:

Chromoionophore

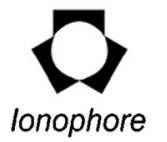

$\mathbf{R}^{-}$

Ion Exchanger chromoionophore simplifies the signal transducing mechanism between the recognition of the target ion and the optical signal output. Several reviews have appeared in the past that emphasized different attributes, such as sensor response mechanism, materials, and potential applications [1, 20-25]. Recently, there has been a growing interest in micrometer- and nanometer-sized ion selective optodes that allow one to apply them to reduced sample volumes. In this review, we highlight recent advances in ionophore-based ion selective optodes associated with their miniaturization and summarize the criteria and possible issues likely to be met to realize and utilize such miniaturized sensors. Other optical sensing platforms, such as molecular probes and functionalized colloidal nanomaterials, are not within the scope of this review.

\section{Ion optode mechanism}

In 1982, Charlton et al. introduced a $\mathrm{K}^{+}$assay where valinomycin-doped test strips were used to detect $\mathrm{K}^{+}$-based on the co-extraction of $\mathrm{K}^{+}$and an anionic dye that was incubated beforehand in diluted samples [26]. This anionic dye, erythrosine $\mathrm{B}$, provided a colorimetric reading for the sensor strip. This formed the first sensor concept that closely resembled modern ion optodes. However, the field of ion optodes did not grow rapidly until 1989, when Morf et al. proposed to add a lipophilic $\mathrm{pH}$ indicator to the sensing phase instead of mixing dye molecules with the sample [27]. This lipophilic $\mathrm{pH}$ indicator was named chromoionophore. The same group subsequently reported neutral carrier-based optodes for ammonium and calcium ions, representing the first successful examples [28, 29].

Today, classic film-based ion optodes contain the following materials: an ionophore, an ion exchanger, a chromoionophore, and a nonreactive hydrophobic polymer or solvent matrix. The actual combination of the materials is dependent on the target ions and can differ from case to case.
Exhaustive combinations for cationic sensor and anionic sensor have been listed in the literature and will not be repeated here [25]. For a cationic optode sensor, such as for $\mathrm{Na}^{+}, \mathrm{K}^{+}$, and $\mathrm{Ca}^{2+}$, the sensing components typically include an electrically neutral chromoionophore, a cation exchanger, and an ionophore, which is mostly neutral as well. To detect anionic species such as $\mathrm{NO}_{2}{ }^{-}, \mathrm{NO}_{3}{ }^{-}, \mathrm{CO}_{3}{ }^{2-}$, and $\mathrm{Cl}^{-}$, a charged chromoionophore, an anion exchanger, and a neutral ionophore are combined. Figure 1 shows the chemical structures of the required sensing components for a $\mathrm{Ca}^{2+}$ selective optode [28] and $\mathrm{CO}_{3}{ }^{2-}$ selective optode [30] as examples.

It is known that cation-selective optodes respond to the ratio of the analyte activity and the proton activity, while anion-responsive optodes respond to the product of the analyte activity and the proton activity [21]. The reason for this is that the chromoionophores used in these sensors made protons inevitably the reference ions in all these sensing schemes. Wolfbeis and co-workers investigated ion optodes modified with polarity sensitive dyes in the absence of chromoionophore [31-33]. This approach resembles Charlton's work in 1982, but the polarity sensitive dyes used are more lipophilic than erythrosine B. Voltage sensitive dyes were recently incorporated in ionophore-based ion selective nanospheres to act as reference ions [34]. In this case, the reference ions are no longer protons but the electrically charged voltage sensitive dyes. The voltage sensitive dye partitions between the organic sensing phase and the aqueous sample phase, the process of which being governed by thermodynamic equilibria.

Fluorescence intensity and absorbance are by far the two major readout modes for ion optodes. However, other techniques, such as luminescence decay time, refractive index, or surface plasmon resonance (SPR) have been successfully applied. For instance, Freiner et al. proposed a waveguide setup where the refractive index of an ion optode film changes upon analyte binding [35]. Suzuki and co-workers reported on a SPR sodium ion sensor using an ion optode membrane film 
Fig. 1 Chemical structures and abbreviations for the sensing components of a $\mathrm{Ca}^{2+}$ selective optode (reference [28]) and a $\mathrm{CO}_{3}{ }^{2-}$ selective optode (reference [30])

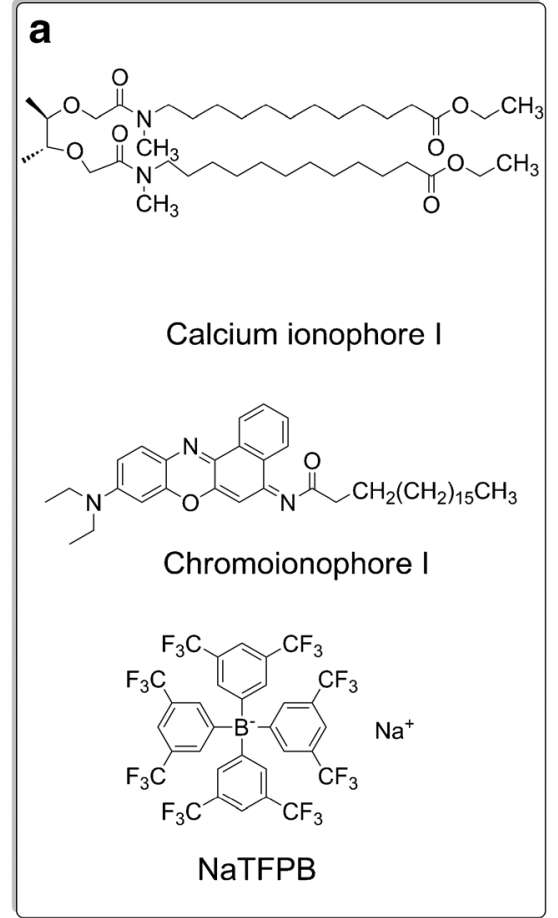

[36], Werner and co-workers presented a potassium ion sensor based on the ion-exchange principle and using fluorescence decay time as readout [37].

\section{Ion selective microspheres and nanospheres}

While film-based ion optodes can be applied to fiber optics [38], flow cells [28], and waveguide devices [39], they are too bulky to operate in a small amount of volume such as living cells. The emergence of miniaturized ion optodes in the form of microspheres and nanospheres can help tackle this challenge. At the same time, the miniaturized particles can be interrogated in a parallel fashion and become potential chemical bio-imaging tools.

Kopelman and co-workers reported on nanosensors that were coined PEBBLEs (probes encapsulated by biologically localized embedding) [40-45]. Using inert, cross-linked acrylamide as a matrix, PEBBLE sensors can incorporate water soluble ion indicators such as rhod-2 and Calcium Green to detect ions without much interference from $\mathrm{pH}$, and has proven to be valuable in the study of a number of intracellular analytes. However, the leaching of the hydrophilic indicator dye remains an issue and must be corrected for in practice. Hydrophobic PEBBLEs have been demonstrated for $\mathrm{K}^{+}$ (Fig. 2a), $\mathrm{NO}_{2}^{-}$, and $\mathrm{Cl}^{-}$and applied in live $\mathrm{C} 6$ glioma cells and rat conceptuses $[43,46]$. The particles exhibited an average diameter from 300 to $700 \mathrm{~nm}$. However, the usable lifetime of the sensors was limited to several hours because of leaching of the sensing materials (i.e., chromoionophore, ion exchanger, and ionophore). Moreover, the preparation was time-consuming and the sensing components had to be adsorbed by the nanospheres after they had been cross-linked. This makes it difficult to produce multicomponent sensor particles with known and optimized compositions.

The situation was improved later on by Clark and coworkers who demonstrated sodium-selective polymeric nanosensors with an average diameter of ca. $120 \mathrm{~nm}$ (Fig. 2b) and a lifetime in solution of roughly 1 wk [47]. The nanospheres were prepared under sonication. The core was made up of classic ion optode materials, whereas the surface was covered with PEG-lipid molecules to offer stabilization. The nanosensors were successfully applied in isolated cardiomyocytes to visualize sodium and potassium flux across cell membrane, as shown in Fig. 3 [48, 49].

The same group also reported on biodegradable optodebased $\mathrm{Na}^{+}$selective nanosensors using polycaprolactone as matrix [50]. The nanosensors were prepared by a solvent displacement method followed by centrifugation and washing. These nanosensors exhibited an average diameter of ca. $260 \mathrm{~nm}$ and lifetime of at least $2 \mathrm{wk}$ at physiological temperature. When in the presence of lipase, the nanosensors degraded within $4 \mathrm{~h}$ at the lipase concentrations found in the liver but still were present after $3 \mathrm{~d}$ at the lipase levels found in serum. Polymer-free nanospheres with an average diameter of ca. $250 \mathrm{~nm}$ were later reported through a similar methodology [51].

In addition, Tohda and Gratzl fabricated ion selective microscopic beads for $\mathrm{Na}^{+}$and $\mathrm{K}^{+}$by a spray drying method [52-54]. Together with microbeads modified with glucose oxidase and those containing just reference dye, the ion 

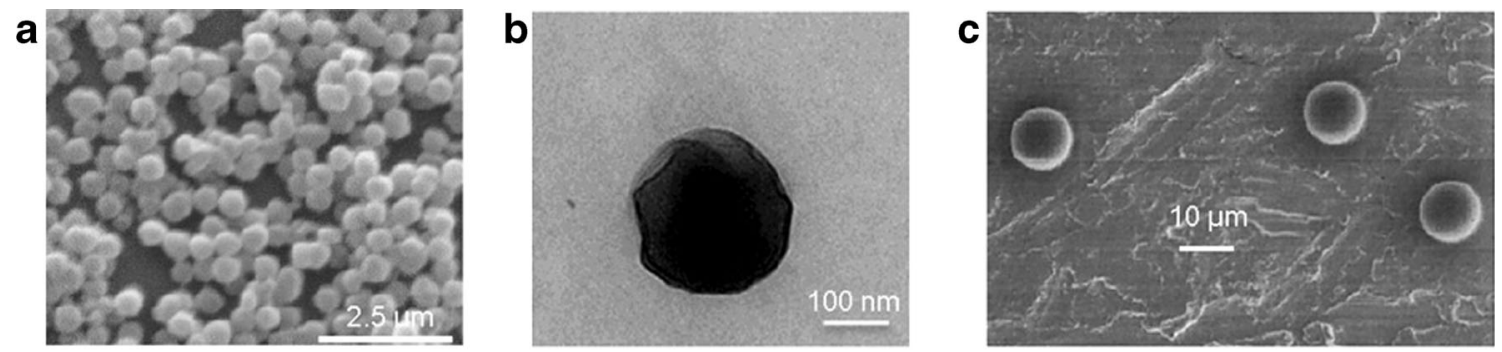

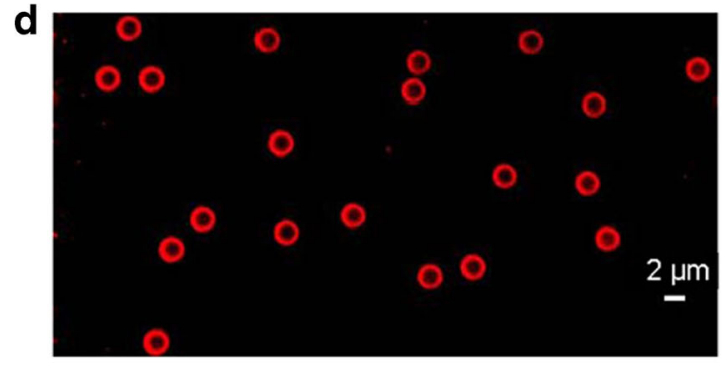

Fig. 2 Microscopic images of different miniaturized ion selective optodes. a SEM image of $\mathrm{K}^{+}$selective PEBBLE sensor adapted from reference [43]; $\mathbf{b}$ TEM image of $\mathrm{Na}^{+}$selective nanosensor adapted from reference [47]; c SEM image of plasticized PVC microspheres adapted

selective beads were assembled into a plastic bar coined "sliver sensor" 100 to $300 \mu \mathrm{m}$ wide and 1 to $1.5 \mathrm{~mm}$ long, and inserted under the skin. It was shown that this optical sensing array could successfully monitor $\mathrm{pH}, \mathrm{Na}^{+}, \mathrm{K}^{+}$, and glucose in the interstitial fluid in vitro. Michalska and coworkers recently reported that ion optode components can be loaded into cross linked poly(maleic anhydride-alt-1octadecene) self-assembled micelles [55]. Carboxylate groups were formed after hydrolysis of the anhydride groups on the copolymer, which could function both as surfactant and ion exchanger. Loading the nanospheres with chromoionophore I alone resulted in a $\mathrm{pH}$ sensitive system.

Earlier, Seitz and co-workers derivatized lightly crosslinked polymer microspheres that swell and shrink as a function of analyte concentration [56]. Ionophores were incorporated into poly(4-hydroxy-3-nitro)styrene microspheres to demonstrate that ion binding accompanied by loss of protons causes the polymer to swell. The particles exhibited diameters from 0.3 to $3 \mu \mathrm{m}$. Although they are not ion optode-based microspheres, the ion exchange process is quite similar to ionophore-based ion selective optodes.

Bakker and co-workers reported on ion selective microspheres that function as film-based optodes and that were prepared using a sonicating particle caster. The microspheres exhibited monodispersity and average diameter on the order of several micrometers (Fig. 2c). Based on this platform, microsensors with selectivity toward $\mathrm{Na}^{+}$[57-59], $\mathrm{Ag}^{+}$[60], $\mathrm{K}^{+}$[57, 61-64], $\mathrm{Ca}^{2+}$ [57], $\mathrm{Pb}^{2+}$ [65], $\mathrm{NO}_{2}^{-}$, [46] $\mathrm{Cl}^{-},[46,57]$, and $\mathrm{PO}_{4}{ }^{3-}$ [66] were successfully fabricated. The multiplexed flow cytometric sensing of blood electrolytes in physiological samples using these fluorescent ion optode microspheres was

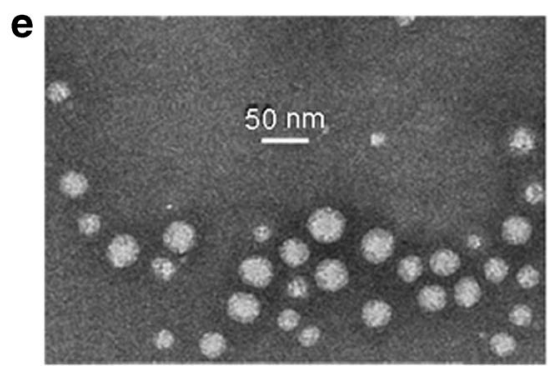

from reference [59]; $\mathbf{d}$ confocal fluorescence image of surface modified polystyrene microparticles adapted from reference [71]; e TEM image of $\mathrm{Na}^{+}$selective nanospheres adapted from reference [72]. Copyright: American Chemical Society

demonstrated [67]. Although PEG was used to stabilize the microspheres, they were still quite prone to coagulation owing to their relatively large size. The excessive amount of aqueous waste generated during the fabrication had to be removed to concentrate the microspheres.

Approaches based on commercial templates were also reported. Quantum dots were incorporated into ion selective optode materials to make both microspheres and nanospheres $[68,69]$. Quantum dots can serve as an additional label for these sensors because they are rather inert. The excitation and emission are tunable by changing their size. They exhibit a wide excitation range, narrow emission bandwidth, and good photostability. Such core-shell structures were made of a quantum dot core, surrounded by a second polymeric coating of the sensing components and a third layer of amphiphilic molecules. At the same time, the fluorescence output may become complicated owing to spectral overlap and possible inner filter and FRET-based energy transfer processes. Silica particles were also reported as template [70]. Since the surface of the commercial silica particles was hydrophilic, silanization was required before doping the core with ion optode sensing components. The particles obeyed optode theory, albeit with rather long response times of ca. $10 \mathrm{~min}$. On the other hand, the surface of nonmodified polystyrene particles is highly hydrophobic and may be utilized to make optode-based microsensors. We recently reported that the simple adsorption of ion exchanger, chromoionophore, and valinomycin on the surface of commercial polystyrene particles resulted in a functional optode sensor [71]. As shown in Fig. 2d, the modification is only limited to the surface because diffusion within polystyrene is extremely slow. 


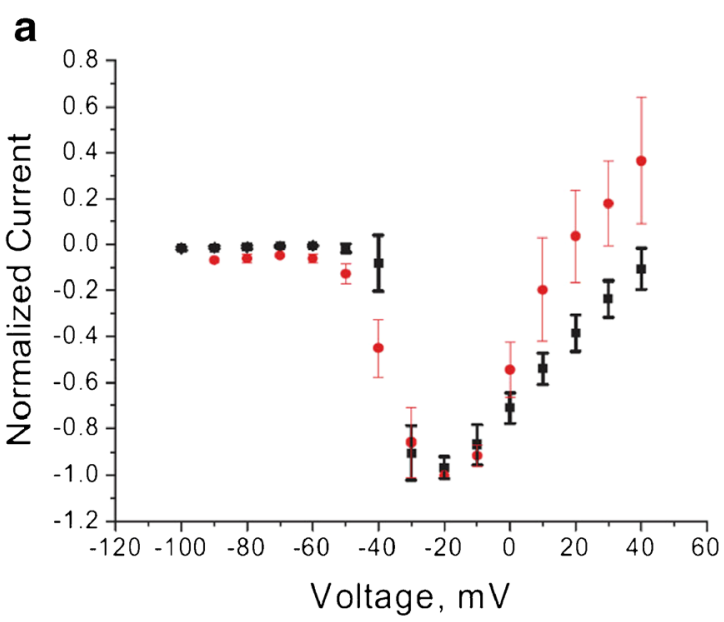

b

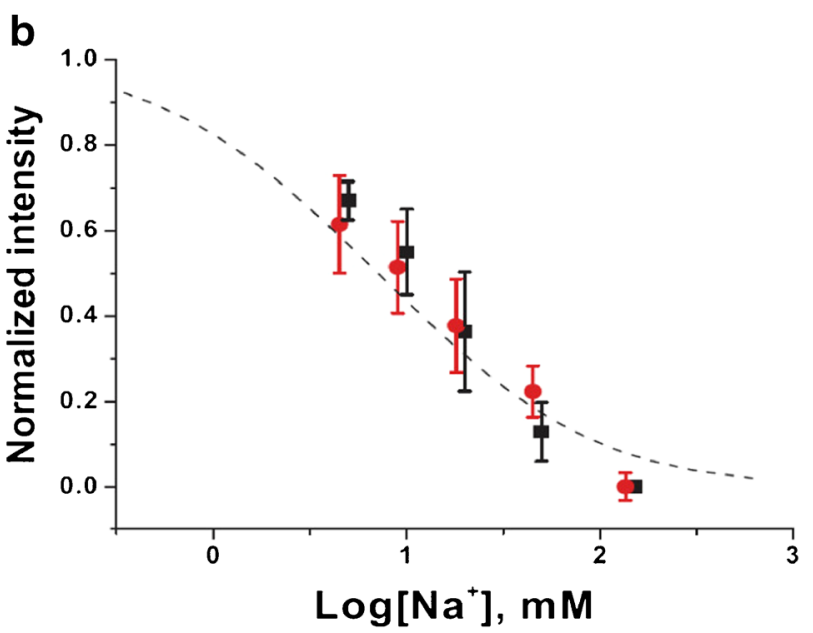

Fig. 3 Intracellular sodium measurements using $\mathrm{Na}^{+}$selective nanosensors from reference [48]. a IV response curve of whole cell patched HEK PN1 cells. Normalized sodium current using control internal filling solution (black squares) and internal filling solution containing sodium nanosensors (red circles). Data represent five

The fabrication of most nanosphere or nanoparticle form ion optodes requires sonication, polymerization, centrifugation, dialysis, or similar procedures. These steps can be timeconsuming and limit the mass production the sensors. Solvent displacement, also called precipitation, is a newly introduced method for the preparation of functionalized nanomaterials. Recently, Xie et al. introduced a new precipitation-based approach to yield ion selective nanospheres, which may alternatively be called nano-emulsions [72]. The fabrication of the nanospheres required one to only dissolve all the sensing components in tetrahydrofuran and subsequent mixing with aqueous solution. The nanospheres could be used immediately after the removal of tetrahydrofuan by purging with compressed air. No further laborious purification steps were required. These nanospheres exhibited typical average diameter of 40 to $100 \mathrm{~nm}$ with good monodispersity (Fig. 2e). It was demonstrated that the nanospheres still behave as film-based optodes. The nanosensors not only functioned as conventional experiments plus standard deviation. b HEK PN1 cell loaded with sodium nanosensors using microinjection. Confocal image showing fluorescence $(633 / 680 \mathrm{~nm})$, bright field, and overlay. c Concentration calibration of nanosensors in vitro (black squares) and in cell-free solution (red circles). Copyright: National Academy of Sciences

ion optodes but also enabled an exhaustive sensing mode that will be discussed below in more detail. The nanospheres were also used to introduce the concept of light controlled ion concentration perturbations [73]. When the chromoionophore is removed, they were also shown to serve as titration reagents that may potentially replace traditional water soluble chelators owing to their much reduced $\mathrm{pH}$ cross-response [74].

\section{Detection mode: equilibrium and exhaustive}

Conventionally, ion selective optodes are interrogated under equilibrium conditions. In other words, the sensing film or particles are in contact with the sample solution to allow for a passive partition of the analyte ions between the aqueous phase and the organic sensing phase until thermodynamic equilibrium is reached. The optical readout, typically absorbance or fluorescence, is recorded to calculate the degree of 
protonation for the chromoionophore, which is then plotted against the logarithm of the analyte concentration (activity, more strictly). A sigmoidal calibration curve is obtained at fixed $\mathrm{pH}$. In addition to passive sensing equilibration, the sensing process also can be triggered by light, as demonstrated by the development of photodynamic ion selective sensors for $\mathrm{Cl}^{-}, \mathrm{Ca}^{2+}$ and $\mathrm{Na}^{+}[6,75,76]$. These sensors are normally doped with photoresponsive compounds such as a spiropyran or a photoacid generator. We refer to the publications cited above for a rigorous discussion of this sensing principle.

To obtain the theoretical ion optode response function, one must consider the phase transfer equilibrium of the extraction process, the charge balance within the sensing phase, the mass conservation and formation constants for the chromoionophore and the ionophore. Detailed derivations have been discussed in the literature. Here, the response function for a cationic analyte $\mathrm{M}^{\mathrm{z}}$ is shown in Eq. 1, where, $a_{M^{2+}}^{a q}$ and $a_{H^{+}}^{a q}$ are the activity of $\mathrm{M}^{\mathrm{z}+}$ and $\mathrm{H}^{+}$in the aqueous sample, $K_{e x}$ is the overall phase extraction equilibrium constant, $\mathrm{n}$ is the complex formation stoichiometry for the ionophore, $\mathrm{R}_{\mathrm{T}}, \operatorname{Ind}_{\mathrm{T}}$ and $\mathrm{L}_{\mathrm{T}}$ are the total concentration of ion exchanger, chromoionophore and ionophore in the sensing phase, $1-\alpha$ is the degree of protonation of the chromoionophore. This response function should apply to both film-based and miniaturized particle-based ion optodes:

$a_{M^{2+}}^{a q}=\frac{1}{z K_{e x}}\left(\frac{a_{H^{+}}^{a q} \alpha}{1-\alpha}\right)^{z} \frac{R_{T}-(1-\alpha) \operatorname{Ind}_{T}}{\left\{L_{T}-z^{-1} n\left[R_{T}-(1-\alpha) \operatorname{Ind}_{T}\right]\right\}^{n}}$

The sensing process is associated with the redistribution of the analyte ions together with hydrogen ions between the sensing phase and the sample phase. For a cationic analyte, ion exchange between protons and the analyte occurs, whereas for an anionic analyte, coexraction of the analyte and protons takes place. Therefore, ion selective optodes, whether film- or particle-based, respond to changes in sample $\mathrm{pH}$ as well. The calibration curve at different $\mathrm{pH}$ values can be very different. For instance, for a calcium selective optode, changing the sample $\mathrm{pH}$ by one unit will shift the sensor response by two orders of magnitude (Fig. 4a). One advantage of this $\mathrm{pH}$ cross response is the ability to tune the sensor response range. For example, a sensor that responds to $\mathrm{Ca}^{2+}$ from $10^{-6} \mathrm{M}$ to $10^{-2} \mathrm{M}$ at $\mathrm{pH} 8$ will do so in the range of $10^{-4} \mathrm{M}$ to $1 \mathrm{M}$ at $\mathrm{pH}$ 7. On the other hand, the $\mathrm{pH}$ cross response is typically regarded as a major drawback of ion selective optodes. In practice, the sample $\mathrm{pH}$ must be controlled or measured at the same time. For sensing carbon dioxide, this problem is avoided by the direct sensing of carbon dioxide activity through the use of a coupled extraction of carbonate and hydrogen ions. This functions in contrast to the Severinghaus $\mathrm{CO}_{2}$ probe, which determines the $\mathrm{CO}_{2}$ level through indirect a
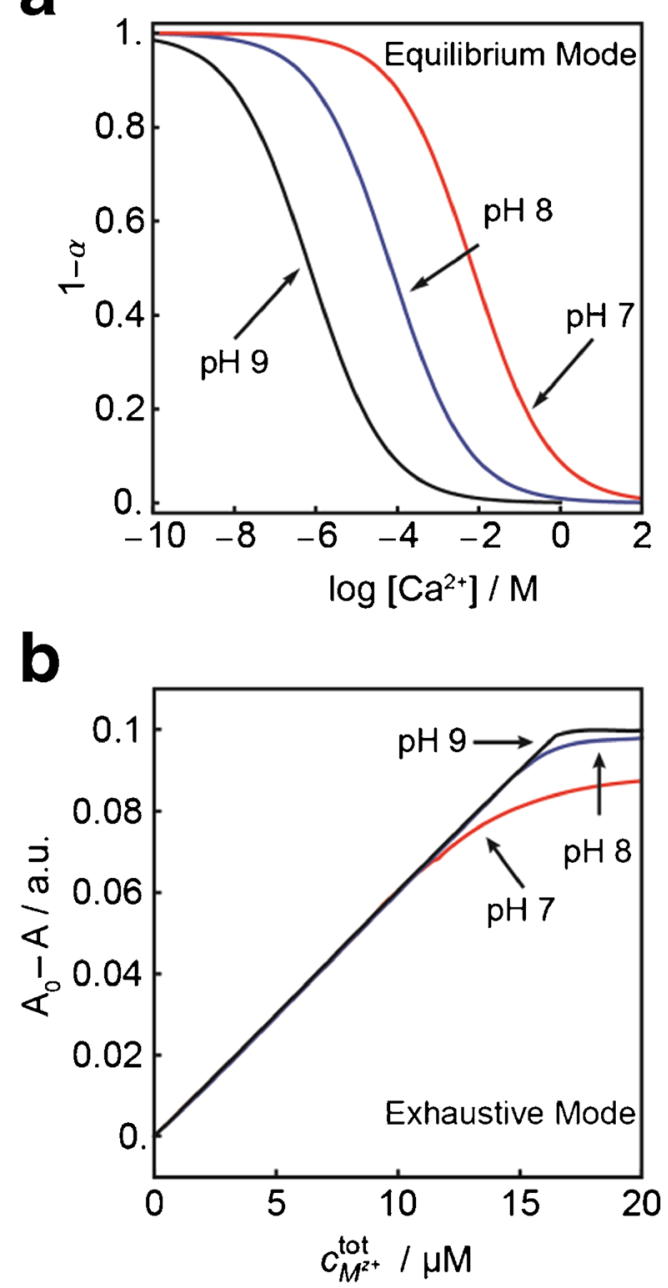

Fig. 4 a Theoretical equilibrium based $\mathrm{Ca}^{2+}$ ion selective optode response at different $\mathrm{pH}$ simulated using Eq. 1; b Theoretical exhaustive mode ion optode response at different $\mathrm{pH}$

measurement of $\mathrm{pH}$ change in aqueous solution upon $\mathrm{CO}_{2}$ extraction [30].

Recently, we have shown that the $\mathrm{pH}$ dependence can be overcome by operating the ion selective sensors in what is called an exhaustive sensing mode [77, 78]. In this case, the sample analyte will ideally be completely consumed by the probes. The response will only depend on the total amount of the analyte and make the exhaustive sensors potentially calibration free. In a classic equilibrium-based sensing mode described by Eq. 1, on the other hand, the sample concentration is usually considered unchanged before and after exposure to the sensor.

The exhaustive detection mode can only work when there are more binding sites in the sensing phase than the amount of analyte in the sample. If a thin optode film is used, a thin layer of sample is preferred since large sample volumes will result in extremely long response times. Using suspensions of nanospheres or microspheres, on the other hand, may avoid this limitation because of a massively 
increased surface to volume ratio and much smaller diffusion distances.

While the analyte is consumed during the exhaustive sensing process, the remaining analyte concentration should still be at equilibrium with the sensor. For the exhaustive mode to be valid, the remaining analyte concentration must be negligible compared with the initial concentration. Based on mass balance and partition equilibria of the analyte, a theoretical model has been put forward for exhaustive sensors based on absorbance measurements, shown in Eq. 2:

$a_{M^{z+}}^{a q}=a_{M^{z+}}^{\overline{a q}}+\frac{1}{z} \frac{A_{0}-A}{\varepsilon b}$

where $a_{M^{z+}}^{\overline{a q}}$ represents the remaining $\mathrm{M}^{\mathrm{z}+}$ activity in the aqueous phase, which follows Eq. $1, \mathrm{~A}_{0}-\mathrm{A}$ is the change in absorbance, $\varepsilon$ is the molar extinction coefficient for the chromoionophore, and $b$ the optical path length for the nanosphere suspension. An important feature of the ion selective optodes working in this mode is that the response is linear with analyte concentration, as shown in Fig. 5 for protamine selective nano-optodes and calcium selective nano-optodes. The linear range is related to the amount of nanospheres used. A wider linear range occurs upon high loading of the nanospheres. Compared with sigmoidal calibrations, a linear calibration curve is statistically much easier to handle.

The exhaustive sensing mode has, of course, its own limitations. In particular, it is not applicable to samples that cannot afford large concentration perturbations. For instance, exhaustive sensors are not suitable for interrogation of ion concentration in live cells because the massive consumption of the ions could potentially kill the cells. Even when the cells can survive such dramatic ion concentration perturbation, the outcome is likely biased because of possible compensation by the cells themselves. An equilibrium sensing mode is preferred in this case since the sample concentration will not be altered.

\section{Response time}

The response time of ion optodes is generally given by the diffusion of all mobile species in the sensing organic phase and the aqueous sample phase. For plasticized PVC films with thicknesses of several micrometers, and with reasonably high sample concentrations, the limiting step is the diffusion of the sensing components within the film. In this case, assuming a mean diffusion coefficient for all mobile species and a film thickness of $d$, the time
Fig. 5 a Absorption spectra of $\mathrm{Ca}^{2+}$ exhaustive nanosensors in response to various $\mathrm{Ca}^{2+}$ concentrations in $10 \mathrm{mM}$ Tris$\mathrm{HCl}$ buffer at $\mathrm{pH} 7.4 ; \mathbf{b}$ Calibration curve for the $\mathrm{Ca}^{2+}$ nanosensors using the absorbance difference at $663 \mathrm{~nm}$ fit with theoretical curve; c Absorption spectra of protamine exhaustive nanosensors in response to various protamine levels in $10 \mathrm{mM}$ tris- $\mathrm{HCl}$ buffer and $0.1 \mathrm{M}$ $\mathrm{NaCl}$ at $\mathrm{pH} 7.4$; d Calibration curve for the protamine nanosensors using the absorbance difference at $641 \mathrm{~nm}$. Adapted from reference [78]. Copyright: American Chemical Society
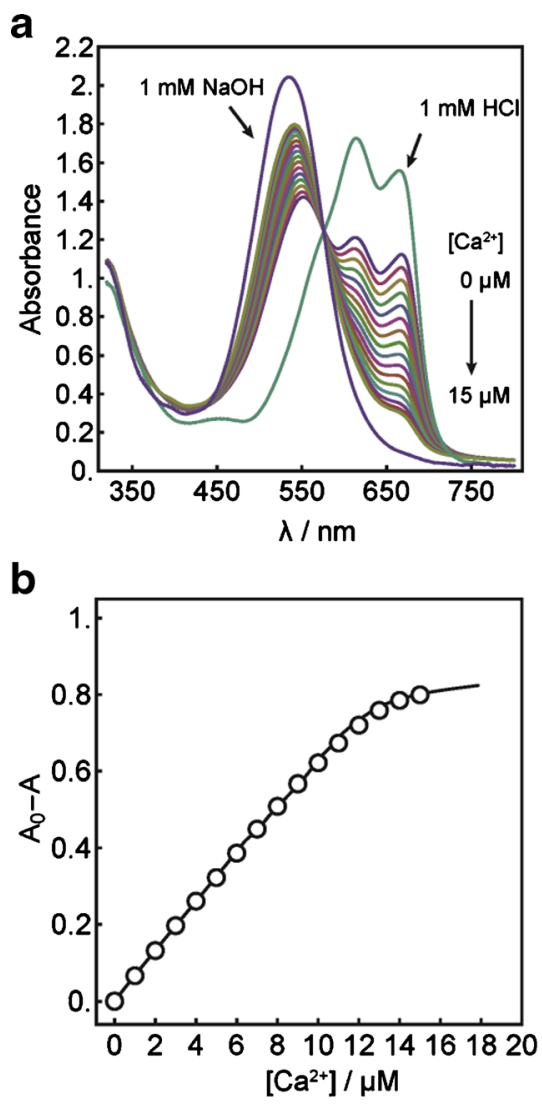
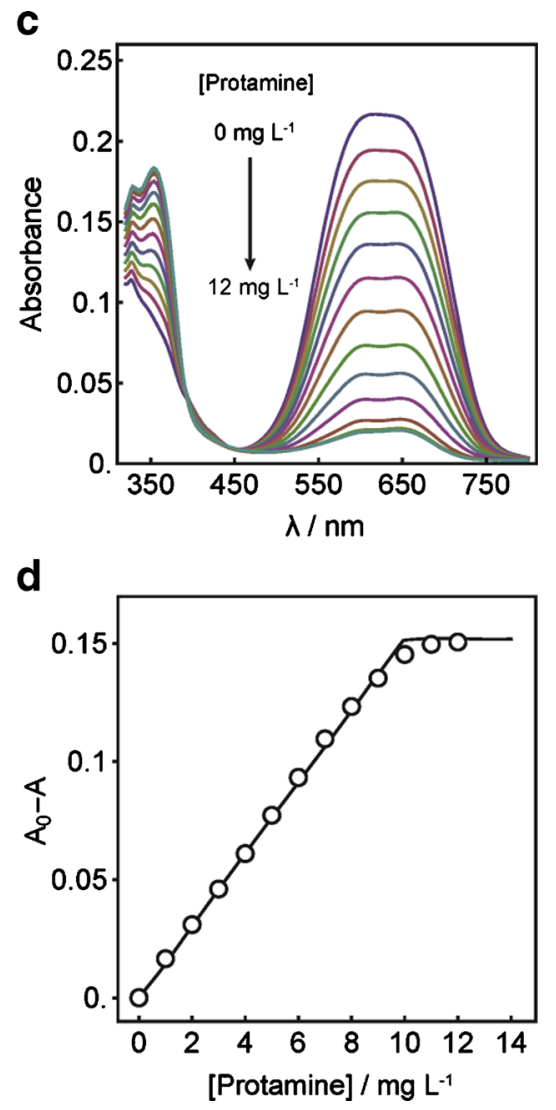
required to achieve $95 \%$ of the steady-state response $t_{95}$ can be simply estimated through Eq. 3 [79]:

$t_{95}=1.13 \frac{d^{2}}{D_{m}}$

where $D_{\mathrm{m}}$ is the diffusion coefficient in the sensing phase. On the other hand, if the analyte level in the aqueous phase is very low, the response time may become limited by mass transport in the aqueous phase. For instance, Lerchi et al. observed earlier that the response time of extremely sensitive optodes for transition metals is mainly dependent on mass transport in solution $[80,81]$. The slow response at low analyte level can be overcome by using ion selective microspheres and nanospheres. As a result of the enhanced surface to volume ratio and spherical diffusion characteristics, mass transport is dramatically accelerated. For instance, Wygladacz et al. reported that for $\mathrm{Ag}^{+}$selective plasticized PVC microspheres with an average diameter of $10 \mu \mathrm{m}$, a response time of less than 15 min was found for $10^{-9} \mathrm{M} \mathrm{AgNO}_{3}$ compared with a response time of $8 \mathrm{~h}$ in the case of film-based optodes [60]. For polyions such as protamine, the diffusion in the organic sensing phase can be quite slow. Previous film-based optical sensors for protamine based on ion exchange principles exhibited response times too long to obtain an equilibrium signal. Xie et al. showed that for protamine selective nanooptodes, the time to reach thermodynamic equilibrium was nearly instantaneous. Moving from a sensing film fixed to a support to freely moving suspended particles dramatically increases the mass transport efficiency [78].

\section{Selectivity}

When ion optodes are miniaturized from the bulk to the nanoscale out of the same material, no dramatic change in the selectivity pattern is expected. The reason for this assumption is that the selectivity for ion optodes mainly depends on the ionophores. Without incorporating ionophores, the selectivity is determined by the thermodynamic equilibrium and in general follows the Hoffmeister series. It has been shown that for PVC-based ion selective microspheres, the selectivity pattern remains similar with film-based optodes based on the same material $[61,64,65] . \mathrm{Na}^{+}$selective nano-optodes incorporating quantum dots have shown similar selectivity for $\mathrm{Na}^{+}$ over $\mathrm{K}^{+}$compared with PVC-based microspheres [69]. The selectivity for the decylmethacrylate-based $\mathrm{K}^{+}$selective PEBBLE over $\mathrm{Na}^{+}$is even slightly better than in plasticized PVC films [43].

However, other factors may influence the selectivity pattern as well. Since the response function is directly derived from fundamental phase transfer and complexation equilibria, different microenvironments will affect the partition equilibrium of the uncomplexed ions. For instance, as shown in Fig. 6, ionophore-free nanospheres reported by Bakker and co-workers showed a similar response to all ions [72]. Similarly, for nanospheres doped with additional ionophores, the response for all interfering ions was found to be nearly the same. These observations indicate that the nanosphere core forms a relatively polar environment compared with filmbased optodes. We speculate that the higher polarity of the core is caused by the insertion of the surfactants, which is more polar than conventional plasticized PVC.

\section{Choice of materials}

\section{Surfactant}

In order to realize micrometer size or nanometer size ion optodes, surfactants may be required to prevent the particles from coagulating. However, the presence of surfactant may also interfere with the sensing process. In particular, ionic surfactants such as sodium dodecyl sulfate (SDS) should be avoided since they may spontaneously extract together with cations into the sensor phase and repel anionic species.
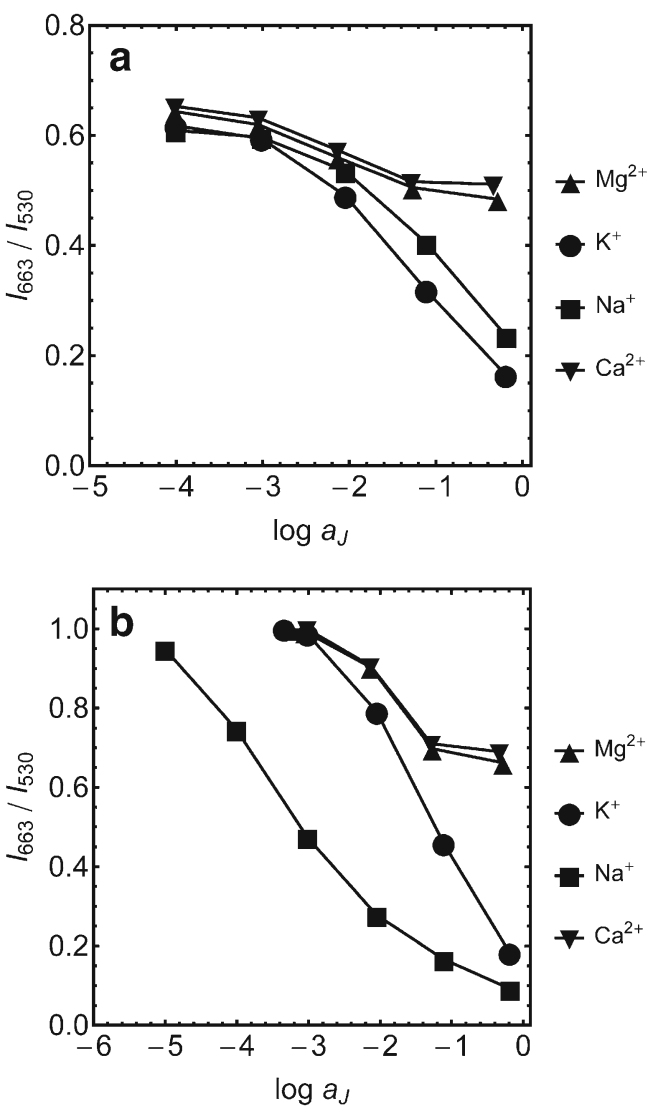

Fig. 6 Selectivity for nanospheres comprised of $\mathrm{CH} 1$ and KTFPB in $10 \mathrm{mM} \mathrm{pH}$ 7.4 Tris-HCl buffer, without (a) and with (b) NaX inside the nanosphere reported in reference [72]. Copyright: American Chemical Society 
Cationic surfactants such as hexadecyltrimethylammonium bromide (CTAB) can repel cations and promote coextraction with anions. Nonionic surfactants, including polyethylene glycol, Pluronic F-127, Triton X-100, Tween 20, and Brij 30 have been used to successfully fabricate ion selective microspheres and nanospheres. These surfactants bear similar hydrophilic groups but differ in the hydrophobic parts. Since the hydrophobic part may insert into a nanoscale organic sensing core, the surfactant is likely to influence the polarity of the particle core and shift the apparent $\mathrm{pKa}$ of the chromoionophore in some cases [72]. Moreover, the hydrophilic chains of these nonionic surfactants are composed of poly(ethylene oxide), which is known to be biocompatible and beneficial for in vivo applications.

\section{Optical reporter}

As for film-based optodes, the choice of chromoionophore for micro/nano-optodes depends on several variables. First, the chromoionophore has to be compatible with the instrumental detection mode. Most chromo-ionophores are suitable for interrogation under absorption mode, established for the family of Nile blue derivatives. They exhibit very high molar extinction coefficients but typically a less attractive quantum yield. Reducing the amount of dye when used in fluorescence mode or imaged under fluorescence microscope may pose a problem.

Recently other compounds have been reported as new chromo-ionophores [82, 83]. However, the palette of chromo-ionophores still needs expansion, especially for those with a high quantum yield. To design a chromoionophore, there are more factors to consider besides quantum yield, such as the $\mathrm{pKa}$ value, spectral range, molar extinction coefficient, photostability, lipophilicity, and so on. Together with the ionophore used, the $\mathrm{pKa}$ value will influence the response range of the sensor. Long wavelength absorption in the visible range and the near infrared region is preferred. Ratiometric dyes are also preferred because they provide a more accurate determination. In addition, the scope of chromoionophore should not be limited to organic dyes. Semiconductor fluorophores, metal ion complexes, and biologically expressed fluorophores are potential candidates as well. These materials easily provide higher quantum yields, better photostability, and narrow absorption and emission bands.

Ion exchanger

The most commonly used ion exchangers are organic analogs based on tetraphenylborate (e.g., tetrakis[3,5bis(trifluoromethyl)phenyl]borate, TFPB) and tetraalkylammonium salts (e.g. tridodecylmethylammonium, TDMA). Others, such as carboxylated poly(vinyl chloride) (PVC) and organic sulphonate, can also serve as ion exchanger but may result in incomplete dissociation because of the relatively high $\mathrm{pKa}$ of these functional groups. Some of the hydrophilic groups of these compounds also can act as amphiphiles when they are used to make nanosensors. Ion exchangers are made very lipophilic to prevent leaching. The leaching from microspheres and nanospheres may be dramatically accelerated because of to the larger surface to volume ratio.

Ionophore

Ionophores help stabilize the target ions and ensure the sensor selectivity. Nowadays, plenty of ionophores are available for cations that include nearly all main group and transition metal ions. For one target ion, one can even find several ionophores with different complex formation constants. Ionophores with higher complex formation constants can shift the sensor response to a lower concentration range and make them more sensitive.

Despite the huge success in developing cation selective ionophores, the advancement of anion selective ionophores is in comparison much less developed. Anions such as $\mathrm{SO}_{4}{ }^{2-}$ and $\mathrm{PO}_{4}{ }^{3-}$ are extremely hydrophilic and thus the complex formation constant for an ionophore must be sufficiently large to compensate the solvation energy difference between aqueous and organic phases. In addition, achieving high specificity is very difficult. Some so-called "anti-crown ether" compounds have been reported to be highly selective to chloride. However, for ions such as nitrate, sulfate, and phosphate, the interference from highly lipophilic anions such as perchlorate and thiocyanate remains a problem. Highly selective ionophores for fluoride have been reported [84]. Recently, salophen-based metal ion complexes have been proposed as ionophores for nitrite, salicylate, copper (II), and monohydrogen phosphate [85-90]. However, these compounds may be susceptible to hydrolysis. Although their lifetime in film-based sensor films can be prolonged by an increase in viscosity and film thickness, when loaded into nanospheres or microspheres, hydrolysis will be accelerated because of the increased surface to volume ratio. In addition, in contrast to most cation selective ionophores that remain optically silent in the visible range, most metal complex-based ionophores do absorb in this range and the spectrum can change upon binding with the anion of interest. This should also be taken into account when designing optical sensors.

\section{Matrix material}

Most film-based ion optodes have adopted plasticized PVC as matrix materials since it is the major material used for ion selective electrodes (ISEs). For miniaturized microsphere and 
nanosphere-based ion optodes, more versatile matrix materials are being explored, including polyacrylamide, polystyrene, polycaprolactone, decyl methacrylate, and polymer-free solvent as described above. The choice of matrix material will help to control the micro/nanosensor size, response time, selectivity, and biocompatibility.

\section{Future perspective}

Despite a history of around 25 years for ion selective optodes, the field of miniaturized ion optodes is still young and rapidly growing. New and exciting concepts and extensions to conventional techniques are regularly introduced into the field, such as different detection modes and various sensor materials. Ion selective optode nanosensors and microsensors have opened new avenues in applied and academic research fields, including imaging in biological environmental samples. In the meantime, some aspects still remain unsatisfactory and require a radical improvement, such as the $\mathrm{pH}$ cross response, relative signal change, brightness, and photostability of the chromoionophore for interrogation in fluorescence mode, and selectivity of anion-specific ionophores. It is also important to discover more convenient and less laborious methods to fabricate miniaturized optode-based sensors. As these characteristics are being improved, ion selective optode sensors will likely become widely accepted and find their way into an increasing number of real-world applications.

Acknowledgments The authors thank the Swiss National Science Foundation (SNF) and the University of Geneva for financial support.

\section{References}

1. Spichiger UE, Freiner D, Bakker E, Rosatzin T, Simon W (1993) Optodes in clinical chemistry: potential and limitations. Sensors Actuators B 11(1/3):263-271

2. Gholivand MB, Babakhanian A, Kaki S, Mohammadi M, Moradi P (2013) A new bulk optical PVC membrane sensor: determination of aluminum in tea leaf, mushroom, potato and Al-Mg syrup samples. Sens Lett 11(9): 1651-1657

3. Meyerhoff ME, Yang VC, Wahr JA, Lee LM, Yun JH, Fu B, Bakker E (1995) Potentiometric polyion sensors: new measurement technology for monitoring blood heparin concentrations during open heart surgery. Clin Chem 41:1355-1356

4. Diamond D, Coyle S, Scarmagnani S, Hayes J (2008) Wireless sensor networks and chemo-/biosensing. Chem Rev 108(2):652-679

5. Hamaguchi Y, Hamaguchi MS, Mohri T (1990) Calcium(2+) measurement using fluorescent indicators. Cell 22(7):258-261

6. Mistlberger G, Xie X, Pawlak M, Crespo GA, Bakker E (2013) Photoresponsive ion extraction/release systems: dynamic ion optodes for calcium and sodium based on photochromic spiropyran. Anal Chem 85(5):2983-2990

7. Bakker E, Qin Y (2006) Electrochemical sensors. Anal Chem 78(12): 3965-3983
8. Akcin N, Koyuncu I, Akcin G (2011) Determination of zinc, nickel and cadmium in natural water samples by flame atomic absorption spectrometry after preconcentration with ion exchange and flotation techniques. Rev Anal Chem 30(2):65-71

9. Li X, Zhou D, Xu J, Chen H (2007) In-channel indirect amperometric detection of heavy metal ions for electrophoresis on a poly(dimethylsiloxane) microchip. Talanta 71(3):1130-1135

10. Alvarez MA, Carrillo G (2012) Simultaneous determination of arsenic, cadmium, copper, chromium, nickel, lead and thallium in total digested sediment samples and available fractions by electrothermal atomization atomic absorption spectroscopy (ET AAS). Talanta 97: $505-512$

11. Aragay G, Pons J, Merkoçi A (2011) Recent trends in macro-, micro-, and nanomaterial-based tools and strategies for heavy-metal detection. Chem Rev 111(5):3433-3458

12. Thakur A, Mandal D, Ghosh S (2013) Sensitive and selective redox, chromogenic, and "turn-on" fluorescent probe for $\mathrm{Pb}(\mathrm{II})$ in aqueous environment. Anal Chem 85(3):1665-1674

13. Town RM, Leeuwen HPV (2004) Depletive stripping chronopotentiometry: a major step forward in electrochemical stripping techniques for metal ion speciation analysis. Electroanalysis 16(6):458-471

14. He Q, Miller EW, Wong AP, Chang CJ (2006) A selective fluorescent sensor for detecting lead in living cells. J Am Chem Soc 128(29): 9316-9317

15. Buccella D, Horowitz JA, Lippard SJ (2011) Understanding zinc quantification with existing and advanced ditopic fluorescent zinpyr sensors. J Am Chem Soc 133(11):4101-4114

16. Mank M, Griesbeck O (2008) Genetically encoded calcium indicators. Chem Rev 108(5):1550-1564

17. Carter KP, Young AM, Palmer AE (2014) Fluorescent sensors for measuring metal ions in living systems. Chem Rev 114(8):4564 4601

18. Han J, Burgess K (2010) Fluorescent indicators for intracellular $\mathrm{pH}$. Chem Rev 110(5):2709-2728

19. Chen T-W, Wardill TJ, Sun Y, Pulver SR, Renninger SL, Baohan A, Schreiter ER, Kerr RA, Orger MB, Jayaraman V, Looger LL, Svoboda K, Kim DS (2013) Ultrasensitive fluorescent proteins for imaging neuronal activity. Nature 499:295-300

20. Seiler K, Simon W (1992) Principles and mechanisms of ionselective optodes. Sensors Actuators B 6(1/3):295-298

21. Bakker E, Bühlmann P, Pretsch E (1997) Carrier-based ion-selective electrodes and bulk optodes. 1. General characteristics. Chem Rev 97(8):3083-3132

22. Spichiger-Keller UE (1997) Ion- and substrate-selective optode membranes and optical detection modes. Sensors Actuators B 38(39):68-77

23. Hisamoto H, Suzuki K (1999) Ion-selective optodes: current developments and future prospects. TrAC Trends Anal Chem 18(8):513524

24. Spichiger U, Simon W, Bakker E, Lerchi M, Biihlmann P, Haug J-P, Kuratli M, Ozawa S, West S (1993) Optical sensors based on neutral carriers. Sensors Actuators B 11:1-8

25. Mistlberger G, Crespo GA, Bakker E (2014) Ionophore-based optical sensors. Annu Rev Anal Chem 7:483-512

26. Charlton SC, Fleming RL, Zipp A (1982) Solid-phase colorimetric determination of potassium. Clin Chem 28(9):1857-1861

27. Morf WE, Seiler K, Lehmann B, Behringer C, Hartman K, Simon W (1989) Carriers for chemical sensors: design features of optical sensors (optodes) based on selective chromoionophores. Pure Appl Chem 61(9):1613-1618

28. Morf WE, Seiler K, Rusterholz B, Simon W (1990) Design of a calcium-selective optode membrane based on neutral ionophores. Anal Chem 62:738-742 
29. Seiler K, Morf WE, Rusterholz B, Simon W (1989) Design and characterization of a novel ammonium ion selective optical sensor based on neutral ionophores. Anal Sci 5:557-561

30. Xie X, Pawlak M, Tercier-Waeber M-L, Bakker E (2012) Direct optical carbon dioxide sensing based on a polymeric film doped with a selective molecular tweezer-type ionophore. Anal Chem 84(7): 3163-3169

31. Wolfbeis OS (1995) Fluorescence-based ion sensing using potentialsensitive dyes. Sensors Actuators B 29:140-147

32. Murkovic I, Lobnik A, Mohr GJ, Wolfbeis OS (1996) Fluorescent potential-sensitive dyes for use in solid state sensors for potassium ion. Anal Chim Acta 334:125-132

33. Huber C, Werner T, Krause C, Wolfbeis OS (1999) Novel chlorideselective optode based on polymer-stabilized emulsions doped with a lipophilic fluorescent polarity-sensitive dye. Analyst 124:1617-1622

34. Xie X, Zhai J, Bakker E (2014) Potentiometric response from ionselective nanospheres with voltage-sensitive dyes. J Am Chem Soc 136(47): 16465-16468

35. Freiner D, Kunz RE, Citterio D, Spichiger UE, Gale MT (1995) Integrated optical sensors based on refractometry of ion-selective membranes. Sensors Actuators B 29:277-285

36. Kurihara K, Nakamura K, Hirayama E, Suzuki K (2002) An absorption-based surface plasmon resonance sensor applied to sodium ion sensing based on an ion-selective optode membrane. Anal Chem 74:6323-6333

37. Krause C, Werner T, Huber C, Klimant I, Wolfbeis OS (1998) Luminescence decay time-based determination of potassium ions. Anal Chem 70:3983-3985

38. Kurihara K, Ohtsu M, Yoshida T, Abe T, Hisamoto H, Suzuki K (1999) Micrometer-sized sodium ion-selective optodes based on a "tailed" neutral ionophore. Anal Chem 71(16):3558-3566

39. Hisamoto H, Kim K-H, Manabe Y, Sasaki K, Minamitani H, Suzuki K (1997) Ion-sensitive and selective active waveguide optodes. Anal Chim Acta 342(1):31-39

40. Clark HA, Hoyer M, Philbert MA, Kopelman R (1999) Optical nanosensors for chemical analysis inside single living cells. 1 . Fabrication, characterization, and methods for intracellular delivery of PEBBLE sensors. Anal Chem 71:4831-4836

41. Clark HA, Kopelman R, Tjalkens R, Philbert MA (1999) Optical nanosensors for chemical analysis inside single living cells. 2 . Sensors for $\mathrm{pH}$ and calcium and the intracellular application of PEBBLE sensors. Anal Chem 71:4837-4843

42. Park EJ, Brasuel M, Behrend C, Philbert MA, Kopelman R (2003) Ratiometric optical PEBBLE nanosensors for real-time magnesium ion concentrations inside viable cells. Anal Chem 75:3784-3791

43. Brasuel M, Kopelman R, Miller TJ, Tjalkens R, Philbert MA (2001) Fluorescent nanosensors for intracellular chemical analysis: decyl methacrylate liquid polymer matrix and ion-exchange-based potassium PEBBLE sensors with real-time application to viable rat C6 glioma cells. Anal Chem 73:2221-2228

44. Si D, Epstein T, Lee Y-EK, Kopelman R (2012) Nanoparticle PEBBLE sensors for quantitative nanomolar imaging of intracellular free calcium ions. Anal Chem 84:978-986

45. Buck SM, Xu H, Brasuel M, Philbert MA, Kopelman R (2004) Nanoscale probes encapsulated by biologically localized embedding (PEBBLEs) for ion sensing and imaging in live cells. Talanta 63:4159

46. Barker SLR, Thorsrud BA, Kopelman R (1998) Nitrite- and chlorideselective fluorescent nano-optodes and in vitro application to rat conceptuses. Anal Chem 70:100-104

47. Dubach JM, Harjes DI, Clark HA (2007) Fluorescent ion-selective nanosensors for intracellular analysis with improved lifetime and size. Nano Lett 7(6):1827-1831

48. Dubach JM, Das S, Rosenzweig A, Clark HA (2009) Visualizing sodium dynamics in isolated cardiomyocytes using fluorescent nanosensors. PNAS 106(38):16145-16150
49. Harjes DI, Dubach JM, Rosenzweig A, Das S, Clark HA (2010) Ionselective optodes measure extracellular potassium flux in excitable cells. Macromol Rapid Commun 31:217-221

50. Balaconis MK, Clark HA (2012) Biodegradable optode-based nanosensors for in vivo monitoring. Anal Chem 84:5787-5793

51. Ruckh TT, Mehta AA, Dubach JM, Clark HA (2013) Polymer-free optode nanosensors for dynamic, reversible, and ratiometric sodium imaging in the physiological range. Sci Rep 3:3366

52. Tohda K, Gratzl M (2003) A microscopic, continous, optical monitor for interstitial electrolytes and glucose. Chem Phys Chem 4:155-160

53. Tohda K, Gratzl M (2006) Micro-miniature autonomous optical sensor array for monitoring ions and metabolites 1: design, fabrication, and data analysis. Anal Sci 22:383-388

54. Tohda K, Gratzl M (2006) Micro-miniature autonomous optical sensor array for monitoring ions and metabolites 2: color responses to $\mathrm{pH}, \mathrm{K}^{+}$and glucose. Anal Sci 22:937-941

55. Kisiel A, Kłucinska K, Głebicka Z, Gniadek M, Maksymiuk K, Michalska A (2014) Alternating polymer micelle nanospheres for optical sensing. Analyst 139:2515-2524

56. Seitz WR, Rooney MTV, Miele EW, Wang H, Kaval N, Zhang L, Doherty S, Milde S, Lenda J (1999) Derivatized, swellable polymer microspheres for chemical transduction. Anal Chim Acta 400:55-64

57. Wygladacz K, Bakker E (2005) Imaging fiber microarray fluorescent ion sensors based on bulk optode microspheres. Anal Chim Acta 532: $61-69$

58. Ngeontae W, Xu C, Ye N, Wygladacz K, Aeungmaitrepirom W, Tuntulani T, Bakker E (2007) Polymerized Nile blue derivatives for plasticizer-free fluorescent ion optode microsphere sensors. Anal Chim Acta 599:124-133

59. Tsagkatakis I, Peper S, Retter R, Bell M, Bakker E (2001) Monodisperse plasticized poly(vinyl chloride) fluorescent microspheres for selective ionophore-based sensing and extraction. Anal Chem 73(24):6083-6087

60. Wygladacz K, Radu A, Xu C, Qin Y, Bakker E (2005) Fiberoptic microsensor array based on fluorescent bulk optode microspheres for the trace analysis of silver ions. Anal Chem 77:4706-4712

61. Tsagkatakis I, Peper S, Bakker E (2001) Spatial and spectral imaging of single micrometer-sized solvent cast fluorescent plasticized poly(vinyl chloride) sensing particles. Anal Chem 73:315-320

62. Ye N, Wygladacz K, Bakker E (2007) Absorbance characterization of microsphere-based ion-selective optodes. Anal Chim Acta 596:195200

63. Peper S, Ceresa A, Qin Y, Bakker E (2003) Plasticizer-free microspheres for ionophore-based sensing and extraction based on a methyl methacrylate-decyl methacrylate copolymer matrix. Anal Chim Acta 500:127-136

64. Retter R, Peper S, Bell M, Tsagkatakis I, Bakker E (2002) Flow cytometric ion detection with plasticized poly(vinyl chloride) microspheres containing selective ionophores. Anal Chem 74:5420-5425

65. Telting-Diaz M, Bakker E (2002) Mass-produced ionophore-based fluorescent microspheres for trace level determination of lead ions. Anal Chem 74:5251-5256

66. Wygladacz K, Qin Y, Wroblewskic W, Bakker E (2008) Phosphateselective fluorescent sensing microspheres based on uranyl salophene ionophores. Anal Chim Acta 614:77-84

67. Xu C, Wygladacz K, Retter R, Bell M, Bakker E (2007) Multiplexed flow cytometric sensing of blood electrolytes in physiological samples using fluorescent bulk optode microspheres. Anal Chem 79: 9505-9512

68. Xu C, Bakker E (2007) Multicolor quantum dot encoding for polymeric particle-based optical ion sensors. Anal Chem 79:3716-3723

69. Dubach JM, Harjes DI, Clark HA (2007) Ion-selective nano-optodes incorporating quantum dots. J Am Chem Soc 129:8418-8419

70. Xu C, Wygladacz K, Qin Y, Retter R, Bell M, Bakker E (2005) Microsphere optical ion sensors based on doped silica gel templates. Anal Chim Acta 537:135-143 
71. Xie X, Crespo GA, Zhai J, Szilágyi I, Bakker E (2014) Potassiumselective optical microsensors based on surface modified polystyrene microspheres. Chem Commun 50:4592-4595

72. Xie X, Mistlberger G, Bakker E (2013) Ultrasmall fluorescent ionexchanging nanospheres containing selective ionophores. Anal Chem 85(20):9932-9938

73. Xie X, Bakker E (2014) Light-controlled reversible release and uptake of potassium ions from ion-exchanging nanospheres. ACS Appl Mater Interfaces 6(4):2666-2670

74. Zhai J, Xie X, Bakker E (2014) Ionophore-based ion-exchange emulsions as novel class of complexometric titration reagents. Chem Commun 50:12659-12661

75. Xie X, Mistlberger G, Bakker E (2012) Reversible photodynamic chloride-selective sensor based on photochromic spiropyran. J Am Chem Soc 134(41):16929-16932

76. Johns VK, Patel PK, Hassett S, Calvo-Marzal P, Qin Y, Chumbimuni-Torres KY (2014) Visible light activated ion sensing using a photoacid polymer for calcium detection. Anal Chem 86(13): 6184-6187

77. Xie X, Zhai J, Bakker E (2014) pH independent nano-optode sensors based on exhaustive ion-selective nanospheres. Anal Chem 86(6): 2853-2856

78. Xie X, Zhai J, Crespo GA, Bakker E (2014) Ionophore-based ionselective optical nanosensors operating in exhaustive sensing mode. Anal Chem 86(17):8770-8775

79. Crank J (1993) The mathematics of diffusion. Oxford University Press, New York

80. Lerchi M, Bakker E, Rusterholz B, Simon W (1992) Lead-selective bulk optodes based on neutral ionophores with subnanomolar detection limits. Anal Chem 64(14):1534-1540

81. Lerchi M, Reitter E, Simon W, Pretsch E, Chowdhury DA, Kamata S (1994) Bulk optodes based on neutral dithiocarbamate ionophores with high selectivity and sensitivity for silver and mercury cations. Anal Chem 66(10):1713-1717

82. Xie X, Crespo GA, Bakker E (2013) Oxazinoindolines as fluorescent $\mathrm{H}^{+}$turn-on chromoionophores for optical and electrochemical ion sensors. Anal Chem 85(15):34-40

83. Xie X, Li X, Ge Y, Qin Y, Chen H-Y (2010) Rhodamine-based ratiometric fluorescent ion-selective bulk optodes. Sensors Actuators B 151(1):71-76

84. Badr IHA, Meyerhoff ME (2005) Highly selective optical fluoride ion sensor with submicromolar detection limit based on aluminum(III) octaethylporphyrin in thin polymeric film. J Am Chem Soc 127:5318-5319

85. Zare HR, Memarzadeh F, Gorji A, Ardakani MM (2005) Iodideselective membrane electrode based on salophen complex of cobalt (III). J Braz Chem Soc 16:571-577

86. Shahrokhian S, Amini MK, Kia R, Tangestaninejad S (2000) Salicylate-selective electrodes based on Al(III) and Sn(IV) salophens. Anal Chem 72(5):956-962

87. Gholivand MB, Niroomandi P, Yarib A, Joshagani M (2005) Characterization of an optical copper sensor based on N, N'bis(salycilidene)-1,2-phenylenediamine. Anal Chim Acta 538(1/2): 225-231

88. Néel B, Asfhar MG, Crespo GA, Pawlak M, Dorokhin D, Bakker E (2014) Nitrite-selective electrode based on cobalt(ii) tert-butylsalophen ionophore. Electroanalysis 26(3):473-480

89. Kim J, Kang DM, Shin SC, Choi MY, Kim J, Lee SS, Kim JS (2008) Functional polyterthiophene-appended uranyl-salophen complex: electropolymerization and ion-selective response for monohydrogen phosphate. Anal Chim Acta 614(1):85-92

90. Ganjali MR, Mizani F, Salavati-Niasari M (2003) Novel monohydrogenphosphate sensor based on vanadyl salophen. Anal Chim Acta 481(1):85-90 